\title{
A Taxonomy in Robot-Assisted Training: Current Trends, Needs and Challenges
}

\author{
Konstantinos Tsiakas \\ HERACLEIA Lab \\ Computer Science and \\ Engineering Department \\ University of Texas at Arlington \\ konstantinos.tsiakas@mavs.uta.edu
}

\author{
Vangelis Karkaletsis \\ Institute of Informatics and \\ Telecommunications \\ National Center for Scientific \\ Research, Demokritos \\ vangelis@iit.demokritos.gr
}

\author{
Fillia Makedon \\ HERACLEIA Lab \\ Computer Science and \\ Engineering Department \\ University of Texas at Arlington \\ makedon@uta.edu
}

\begin{abstract}
In this paper, we present a taxonomy in Robot-Assisted Training; a growing body of research in Human-Robot Interaction which focuses on how robotic agents and devices can be used to enhance user's performance during a cognitive or physical training task. The proposed taxonomy includes a set of parameters that characterize such systems, in order to highlight the current research trends and needs for the design, development and evaluation of Robot-Assisted Training systems. Towards this direction, we review related taxonomies in Human Robot Interaction, as well as recent works and applications in Robot-Assisted Training. The motivation of this research is to identify and discuss issues and challenges, focusing on the personalization aspects of a Robot-Assisted Training system.
\end{abstract}

\section{CCS Concepts}

•Human-centered computing $\rightarrow$ Human computer interaction (HCI); Interaction design;

\section{Keywords}

Robot Assisted Training (RAT), Human Robot Interaction (HRI), Assistive Robotics, Personalization

\section{INTRODUCTION}

Robot-Assisted Training (RAT) is a growing body of research in Human-Robot Interaction (HRI) which studies how robots can assist humans in a task-dependent interaction. RAT systems have a wide range of applications, varying from physical assistance in post-stroke rehabilitation and robotic prosthetics [13, 32], cognitive training for patients suffering from dementia and Alzheimer's disease [4, 19], to intervention and therapy for children with Autism Spectrum Disorders [29, 11, 22] and Socially Assistive Robotics (SAR) for language learning and children education $[23,16,9]$. As

Permission to make digital or hard copies of all or part of this work for personal or classroom use is granted without fee provided that copies are not made or distributed for profit or commercial advantage and that copies bear this notice and the full citation on the first page. Copyrights for components of this work owned by others than ACM must be honored. Abstracting with credit is permitted. To copy otherwise, or republish, to post on servers or to redistribute to lists, requires prior specific permission and/or a fee. Request permissions from permissions@ acm.org.

PETRA '18, June 26-29, 2018, Corfu, Greece

(C) 2018 ACM. ISBN 978-1-4503-6390-7/18/06 .. \$15.00

DOI: https://doi.org/10.1145/3197768.3197787 a multidisciplinary research field, it requires expertise in several research areas, including robotics, human-machine interaction, machine learning, data mining, computer vision, as well as expertise in psychology and educational sciences, kinesiology, occupational therapy and others.

Despite this large variety of applications, target populations and system requirements, a common goal of RobotAssisted Training systems is to enhance user's performance by providing personalized and targeted assistance towards maximizing training and learning effects. Personalization has the potential to create a tailored and compelling experience that encourages and assists users to perform a given task and meet the training goals.

The motivation and purpose of this research is to identify a common set of parameters (i.e., taxonomy categories) that characterize a Robot-Assisted Training system, taking into consideration related taxonomies in human robot interaction, as well as recent works in Robot-Assisted Training systems, in order to highlight the current research trends and challenges in this growing research area.

\section{RELATED TAXONOMIES IN HRI}

One of the most generalized and broad classifications for HRI systems provides a classification framework based on eleven taxonomy categories [36, 35]: task type, task criticality, robot morphology, ratio of people to robots, composition of robot teams, level of shared interaction among teams, interaction roles, physical proximity, decision support for operators, time-space taxonomy and autonomy level/amount of interventions from operators.

These different variables can be used to define and classify an HRI system. System requirements can be defined by task type, task criticality and robot morphology. The task type variable defines the task in a high-level representation (e.g., physical rehabilitation task). It is important because it sets the system requirements and the basic design guidelines. Some possible values of this variable are: tutoring session, assembly manufacturing task, rehabilitation exercises, etc. Task criticality considers safety issues (e.g., human safety risk) and has three values: high, medium, low. Since robot appearance affects how people interact with it, the robot morphology variable describes the robot appearance type, i.e., anthropomorphic, zoomorphic, and functional.

Depending on the application, there are different interaction types and roles for human and robot members. One parameter under this category is the ratio of people to robots, which simply defines the number of humans and 
robots participating in the interaction. Another parameter is the type of interaction between human and robot participants, defining the level of shared interaction among (robot and human) teams (as we show in Figure 3). The most straightforward example is a single robotic agent that interacts with a single human user. A more complex example is a human operator that sends commands to a team of robots, which has to autonomously coordinate its members to execute the command. Another example is a team of human users that coordinates and sends specific commands to independent robots.

Since human participation is essential for any HRI system, human roles must be well-defined. Scholtz [30] has defined five different roles for a human participant in an interaction with a robot: supervisor, operator, teammate, mechanic/programmer and bystander. Moreover, two more are added by Goodrich [14]: mentor and information consumer. In many applications, where the human acts as an operator or supervisor, an HRI system should provide the user with decision support. The human user needs to monitor, intervene, and modify robot's behavior, when needed. Providing appropriate information to the operator can enhance their decision making. For example, the robot can visualize information about the list of all available sensors and data streams. Interactive methods can be used to make the system's decision process transparent to the user, as humans and machines require shared awareness and shared intent during human-robot interactions [25, 12]. Another defining factor for HRI is the level of autonomy (or the amount of human intervention). Human operators or supervisors often have the ability to control the robot and modify its behavior. The level of the autonomy is defined as the amount of time that the robot acts in an autonomous manner. In many cases, this value can be adjusted during the interaction, resulting to a progressively autonomous system. Human workload and cognitive capacity are two important factors to take into consideration in order to define the level of autonomy.

Other parameters that are defined by this taxonomy are spatiotemporal and define human robot interaction in terms of space and time. More specifically, this parameters categorize an HRI system based on whether human and robot share the same space (collocated, non-collocated), and whether they act at the same time or not (synchronous, asynchronous). Moreover, in a collocated HRI system, the robot can be defined by different proximity behaviors e.g., avoiding, passing, following, approaching, touching, and/or none. Focusing on specific applications and domains requires a more detailed description. For example, SAR systems have been used for physical rehabilitation [34], where proxemics are defined based on social interaction zones (e.g., social, personal, intimate) used to define robot's personality (e.g., introvert, extrovert).

Depending on the application and the system requirements, several taxonomies have been introduced for human robot interaction systems, such as human-robot collaboration, child-robot interaction, assistive robotics and others. More specifically, Salter [28] presented a taxonomy for childrobot interaction (CRI), based on the control factors for both robots and participants. They used three categories for both robots and human participants: Autonomy, Group and Environment. For example, the robotic autonomy (RA) can be classified as one of the following: autonomous, fixed, combination, Wizard of $\mathrm{Oz}$, and remote-controlled. The par- ticipant autonomy (PA) can be: free, natural, comfortable, directed, and controlled, based on how the users are allowed to interact with the robot. The authors have provided a taxonomy rating in relation to participant and robot influences, for all three categories. They used a rating scale from 1 (None) to 9 (High) to describe the level of control of robots and participants.

Other taxonomies focus and elaborate on specific parameters, such as robot autonomy level. In [3], the authors present a framework for Levels Of Robot Autonomy (LORA) in HRI, identifying parameters that influence and get influenced by the level of robot autonomy. They provide a guideline flow chart to determine robot autonomy and effects on HRI. Their taxonomy for robot autonomy takes into consideration the level of autonomy during sensing, planning and acting. The guidelines can be used to identify task and environmental influences on robot autonomy level, measure and categorize autonomy level and identify HRI parameters that have an impact on robot autonomy.

Focusing on human robot collaboration systems, another recent taxonomy describes the level of automation, specifically for collaborative robots [7]. The Interaction Readiness Model (IRM) classifies a system in one of the four levels, based on the level of automation. This model correlates the level of automation with task complexity in a manufacturing environment. The automation level varies from gated robots mode, where robot is idle while human is present to fully interactive mode, where humans and robots learn how to solve a synergistic task. This model has been defined based on real industrial needs, towards Industry 4.0 and "robofacturing" [18].

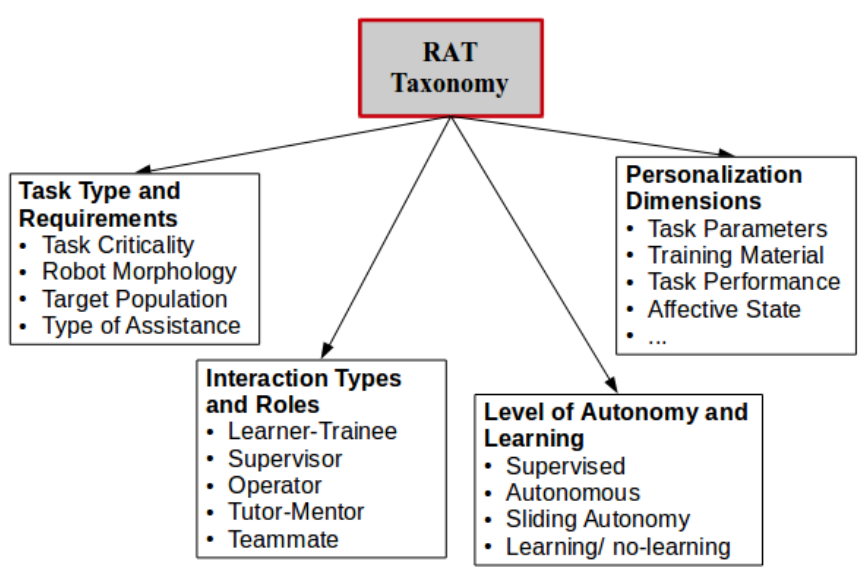

Figure 1: Taxonomy Categories for Robot-Assisted Training

\section{TAXONOMY CATEGORIES}

Based on the existing taxonomies and classification frameworks, we propose a list of parameters that may be considered for the design and development of a Robot-Assisted Training system, as we show in Figure 1. The defined categories are: Task Type and Requirements, Interaction Types and Roles, Level of Autonomy and Learning and Personalization Dimensions. We argue that these categories should be defined in the order presented, since the values of some parameters are dependent to others, i.e., the 
requirements of a rehabilitation system (high level of task criticality) may require a supervisor to monitor the interaction - interaction roles. For each category, we review recent works in order to highlight the current trends; an indicative set of examples is shown in Figure 2.

\subsection{Task Type and Requirements}

When designing a Robot-Assisted Training system, the task type and requirements are the first parameters to be defined, since they can set the tone for the overall design, implementation and evaluation process. The task type and requirements define important parameters as task criticality and safety issues, target populations, robot morphology, set of appropriate sensors and type of assistance (physical, social, mixed). Task type provides a high-level description of the task and the system requirements. Based on a recent taxonomy [37], types of assistive robots include physically assistive robotics (PAR), socially assistive robotics (SAR), as well as sensory and feedback systems.

For example, in [15] the authors have presented a SAR system for language learning with children, where the system uses a camera to capture and analyze facial expressions and affective features (gaze, smile, engagement, valence, etc) in order to provide a personalized affective interaction through social verbal behavior (valence and engagement of spoken instructions). Another work presents a physically assistive robot for upper-limb rehabilitation [20]. In this work, the authors presented an automated system for a rehabilitation robotic (physically assistive) device that guides stroke patients through an upper-limb reaching task. The system uses task-related observations (e.g., task completion time and assistance needed) to estimate user-related metrics (e.g., user fatigue, progress, etc.) and adapt the reaching task parameters to enhance training effects. As part of the system's requirements, the authors argue that the use of sensors (camera, EMG sensors, etc.) could lead to noisy and untrustworthy observations and system's decisions. Due to high task criticality, a supervisor monitors the system's decisions and intervenes when needed.

Social robots can provide supportive behavior, feedback and recommendations, as well as attention acquisition systems to assist users in several applications, e.g., attention acquisition through gestures in a memory game [17]. Another example demonstrates how socially assistive robots can be deployed for physical rehabilitation [34], investigating different robot behavior parameters (human-robot personality matching, robot proxemics, etc.). Social assistance can also improve compliance and performance for physical exercising in child-human interaction [26].

\subsection{Interaction Types and Roles}

Similar to previous taxonomies, we define the humanrobot interaction types and roles. These parameters define the interaction types; how the human-robot team is formulated and communicates, as well as the interaction roles for each part of the interaction. In Figure 3, we show the different interaction types of humans and robots in a RobotAssisted Training system.

Previous taxonomies have focused on human members roles [36, 35], as mentioned in Section 2. In this work, we focus on interaction roles that both human and robot members can have in a Robot-Assisted Training session, including: primary user, supervisor, operator, instructor and teammate. A primary user is the end user who participates actively in the interaction (e.g., patient). While the most frequent case is that this is a human user, there are works that focus on training a secondary user (therapist) [1], by simulating the primary user using the robot in order to evaluate the system from the aspect of the supervisor [31]. A (human or robot) supervisor monitors the training session (i.e., through sensors or interfaces) to capture essential information of the training session (e.g., task parameters, user performance and condition, etc.). A human operator can control the parameters of the training session (e.g., using a control interface), while a robot operator is the actual actuator which assists the user in the task (e.g., robotic arm for wrist rehabilitation [2]). An instructor plays the role of a tutor who guides and instructs the user during the task (e.g., educational robots). Team co-ordination and collaboration can be used as training tasks, thus the role of a (human or robot) teammate who interacts with the user can be an important member role in a training session.

\subsection{Level of Autonomy and Learning}

An essential aspect of a Robot-Assisted Training system is the level of robot autonomy, which defines whether the robot acts autonomously or under the guidance of a human user. Specific system requirements and parameters may require the presence of a human expert who acts as a supervisor to ensure safety and efficiency during the training session. Influenced by LORA [3], the level of autonomy in a RAT system varies from tele-operation to fully-autonomous systems, including sliding autonomy systems.

In the upper-limb reaching task example (Section 3.1), the system suggests an action to the supervisor, through a GUI, and the supervisor agrees or disagrees with the system decision, resulting to a safe semi-autonomous interaction. This Wizard-of-Oz (WoZ) paradigm has been extensively used for RAT applications, where the robot executes the behaviors decided by a human supervisor. Despite its effectiveness, a main limitation relates to the amount of expert workload and attention to ensure a safe robot behavior. Towards this end, recent approaches enable the robot to learn through human (expert) input and progressively act in an autonomous manner. Robotic agents can be either learning or non-learning agents, or they can switch between these levels of learning, depending on different parameters (i.e., uncertainty). Active Learning is a research area which studies when an agent should ask for human input (i.e., correct label/action) in order to improve system performance. Interactive Machine Learning and Interactive Reinforcement Learning are two promising approaches to integrate such human expertise and feedback in the learning mechanism of an interactive system (Human-in-the-Loop). Following such interactive learning approaches, intelligent WoZ interfaces can enable an assistive robot to integrate expert knowledge and guidance and switch from tele-operation to a progressively autonomous mode, decreasing expert workload and effort.

For example, neural networks have been used to learn robot behavior from human expert input in a RAT session [31]. The presented system simulates a RAT session, where a human supervisor monitors a robot-child and a robotinstructor during a card classification task, using a WoZ interface. The neural network is trained using human input as training labels. Their user study results indicate that 


\begin{tabular}{|c|c|c|c|}
\hline Task Type and Requirements & Interaction Types and Roles & Level of Autonomy and Learning & Personalization Dimension(s) \\
\hline $\begin{array}{c}\text { Socially Assistive Robotics (SAR) } \\
\text { for Language Learning in Children } \\
\text { Education [15] }\end{array}$ & $\begin{array}{c}\text { A social robot acts as an affective tutor } \\
\text { as the child plays a language learning } \\
\text { game }\end{array}$ & $\begin{array}{l}\text { The robot acts fully autonomously and } \\
\text { adjusts its behavior online using } \\
\text { Reinforcement Learning (RL) }\end{array}$ & $\begin{array}{c}\text { The robot personalizes its affective strategy } \\
\text { by adjusting its engagement and valence } \\
\text { during verbal instructions }\end{array}$ \\
\hline $\begin{array}{l}\text { Socially Assistive Robot for Post } \\
\text { Stroke Rehabilitation Therapy for } \\
\text { Elderly Patients [34] }\end{array}$ & $\begin{array}{l}\text { The robot acts as a therapist who } \\
\text { monitors, assists, encourages users } \\
\text { during rehabilitation }\end{array}$ & $\begin{array}{c}\text { The robot acts fully autonomously and } \\
\text { learns the optimal personalized policy } \\
\text { using policy gradient RL }\end{array}$ & $\begin{array}{l}\text { The robot adjusts its personality (e.g., } \\
\text { introversion/extroversion), as well as } \\
\text { movement parameters (e.g., proxemics) }\end{array}$ \\
\hline $\begin{array}{c}\text { Robot-Based Wrist Rehabilitation } \\
\text { through Gamification and Dynamic } \\
\text { Player Modeling [2] }\end{array}$ & $\begin{array}{l}\text { The user performs a game-based } \\
\text { rehabilitation task using a robotic } \\
\text { haptic device }\end{array}$ & $\begin{array}{l}\text { The robot acts autonomously and learns } \\
\text { through dynamic player modeling and RL }\end{array}$ & $\begin{array}{c}\text { The system adjusts the difficulty of the game } \\
\text { by modifying the game parameters to } \\
\text { challenge the user }\end{array}$ \\
\hline $\begin{array}{c}\text { Adaptive Upper-Limb Stroke } \\
\text { Rehabilitation using a Robotic Arm } \\
\text { for a Reaching Task [20] }\end{array}$ & $\begin{array}{l}\text { The robotic arm trains the user in a } \\
\text { reaching task. A human supervisor } \\
\text { monitors system's decisions }\end{array}$ & $\begin{array}{l}\text { The robot act autonomously based on a } \\
\text { given policy (no learning); an expert } \\
\text { evaluates the chosen action }\end{array}$ & $\begin{array}{l}\text { The system adjusts the task difficulty by } \\
\text { choosing the reaching target, the level of } \\
\text { resistance, and when the exercise should stop }\end{array}$ \\
\hline $\begin{array}{c}\text { Social Robot Assistant for } \\
\text { Attention Acquisition during a } \\
\text { Memory Game [17] }\end{array}$ & $\begin{array}{l}\text { The robot acts as a tutor who guides } \\
\text { user's attention during a memory } \\
\text { game, in a wizarded setup (e.g., } \\
\text { supervisor) }\end{array}$ & $\begin{array}{l}\text { The system acts semi-autonomously. The } \\
\text { supervisor provides the user state and RL } \\
\text { selects appropriate gestures }\end{array}$ & $\begin{array}{l}\text { The robot provides personalized behavior by } \\
\text { selecting the appropriate combinations of } \\
\text { gestures to grab user attention }\end{array}$ \\
\hline $\begin{array}{l}\text { Physical Exercising for Children } \\
\text { using a Social Robot and Wizard- } \\
\text { of-Oz Interfaces [26] }\end{array}$ & $\begin{array}{c}\text { The robot demonstrates the exercises } \\
\text { to be performed. A supervisor controls } \\
\text { the robot through a WoZ interface }\end{array}$ & $\begin{array}{c}\text { The system acts in a semi-autonomous } \\
\text { manner. A supervisor controls the robot } \\
\text { (WoZ). The robot learns from human input }\end{array}$ & $\begin{array}{c}\text { The robot personalizes the exercise regimen } \\
\text { and its behavior according to exercise } \\
\text { performance and compliance }\end{array}$ \\
\hline
\end{tabular}

Figure 2: Recent works in Robot-Assisted Training. Considering our proposed taxonomy, we characterize these systems based on (a) Task Type and Requirements, (b) Interaction Types and Roles, (c) Level of Autonomy and Learning and (d) Personalization Dimensions

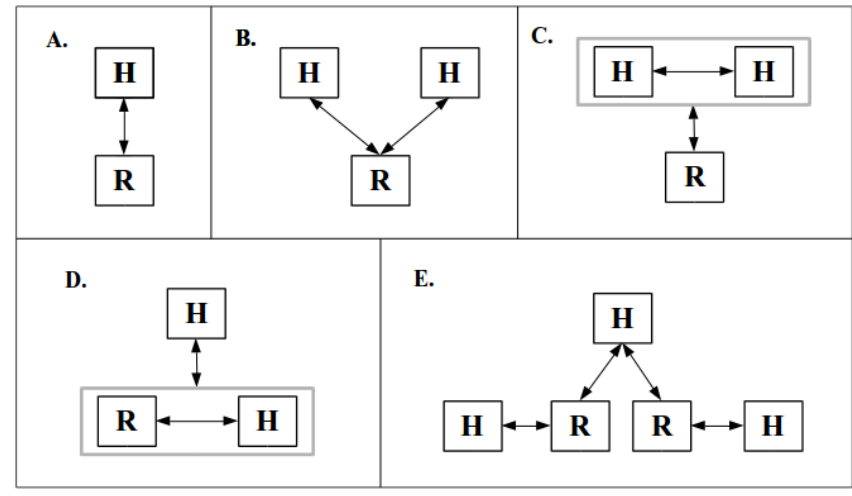

Figure 3: Examples of Interaction Types in RobotAssisted Training (inspired by [35]).

learning agents can decrease expert workload, as they learn how to provide human-like decisions. The robot shifts from a tele-operated agent (WoZ) to a fully autonomous robot, demonstrating that progressive robot autonomy results in lower supervisor workload.

\subsection{Personalization Dimensions}

Personalization plays an integral role in designing an efficient Robot-Assisted Training system. Based on the famous Bloom's 2 sigma problem [5], one-to-one tutoring presents better learning effects than group (conventional) tutoring. Parameters that affect efficiency include training material (e.g., exercise regimen) and teacher behavior (e.g., supportive, challenging, etc.). Such parameters can be adjusted in order to maximize tutoring/training effects for each individual. Depending on the system parameters defined by the other taxonomy categories, we define the personalization dimensions.

Personalization dimensions refer to (a) the set of observations that the system perceives and considers in order to adjust its behavior and (b) the set of control parameters, that are adjusted to achieve personalization, as we show in Table 1.

Table 1: Personalization Dimensions. A list of observations and control parameters for personalization

\begin{tabular}{|c|c|}
\hline \multicolumn{2}{|c|}{ Personalization Dimensions } \\
\hline observations & control parameters \\
\hline task performance & task difficulty \\
user attention & task duration \\
affective state & supportive behavior \\
response time & haptic feedback \\
user progress & training material \\
errors & robot proxemics \\
\hline
\end{tabular}

One of the research questions regarding personalization is how to use these observed parameters in order to learn the control parameters. Interactive Reinforcement Learning (IRL) techniques have been used to facilitate robot learning from human-generated feedback. For example, a robot that learns behavior by utilizing the emotional (and other social) signals of the user could facilitate real-time personalization in human-robot interaction on the wild.

For example, the affective language tutor [15] described in Figure 1, uses a facial expression and feature extraction software in order to estimate child's affective state (engagement and valence). The system combines these estimated values into a reward signal and the system learns to adjust its be- 
havior by selecting appropriate motivational strategies (using verbal and non-verbal actions), based on current child's state (affect and performance). However, it is of high importance to ensure a user-friendly and non-intrusive sensory system, selecting the appropriate (optimal) set of sensors, considering factors as usability, effectiveness and efficiency.

\section{CONCLUDING REMARKS}

In this paper, we presented a taxonomy in Robot-Assisted Training, considering related taxonomies in Human-Robot Interaction, as well as current research trends and needs in this growing body of research. The purpose of this taxonomy is to highlight several research objectives related to Robot-Assisted Training systems. We presented a systematic literature review, aiming to delineate different aspects and trends to be taken into consideration when designing a RAT-based system. Future improvements, updates and additions are required to establish a well-defined taxonomy in such a wide research/application area, focusing on the needs and dimensions for personalization. The motivation of this research is how different types of users can participate in the personalization procedure.

Robot-Assisted Training systems usually operate in contextually rich environments that can provide the system with valuable information to achieve personalization. A research question that arises is how to identify the optimal (e.g., minimum) set of modalities and sensors to ensure an efficient and effective interaction. As we discussed in Section 3.2, different interaction types and member roles result to different types of human feedback that can be captured by different sensors/interfaces including, cameras, microphones, EEG sensors, GUIs, joysticks, and many others.

These different types of feedback can be integrated to the system's personalization mechanism, using interactive machine learning methods, towards interactive personalization. Interactive Machine Learning can utilize human-generated feedback (i.e., facial expressions, emotion, GUI input, etc.) in order to facilitate personalization in the wild, covering also rare user cases (e.g., unobserved or outlier users). Research works investigate how informative user interfaces and interactive learning methods can increase user engagement while interacting with a learning control interface [24].

Personalization is a complex computational problem that requires the training agent to interactively assess, adapt, and leverage a model of the user's ability and needs [6] and can benefit from research reviews in several areas, including but not limited to, Intelligent Tutoring Systems [21], Student Modeling [8], Affective Computing [33], Cyber-Physical Systems [27] and Machine Learning for Interactive Systems and Robots [10].

\section{ACKNOWLEDGMENTS}

This work is based upon research supported by NSF under award numbers NSF-CHS 1565328, NSF-PFI 1719031 and by the educational program of NCSR Demokritos in collaboration with the University of Texas at Arlington.

\section{REFERENCES}

[1] O. A. Alsos and D. Svanæs. Designing for the secondary user experience. In IFIP Conference on Human-Computer Interaction, pages 84-91. Springer, 2011.
[2] K. Andrade, G. Fernandes, G. Caurin, A. Siqueira, R. Romero, and R. Pereira. Dynamic player modelling in serious games applied to rehabilitation robotics. In SBR-LARS Robotics Symposium and Robocontrol, pages 211-216. IEEE, 2014.

[3] J. Beer, A. D. Fisk, and W. A. Rogers. Toward a framework for levels of robot autonomy in human-robot interaction. Journal of Human-Robot Interaction, 3(2):74, 2014.

[4] V. Bernabei, D. De Ronchi, T. La Ferla, F. Moretti, L. Tonelli, B. Ferrari, M. Forlani, and A. Atti. Animal-assisted interventions for elderly patients affected by dementia or psychiatric disorders: a review. Journal of psychiatric research, 47(6):762-773, 2013.

[5] B. S. Bloom. The 2 sigma problem: The search for methods of group instruction as effective as one-to-one tutoring. Educational researcher, 13(6):4-16, 1984.

[6] J. Canny. Interactive machine learning. University of California, Berkeley, 2014.

[7] L. G. Christiernin. How to describe interaction with a collaborative robot. In Proceedings of the Companion of the 2017 ACM/IEEE International Conference on Human-Robot Interaction, pages 93-94. ACM, 2017.

[8] K. Chrysafiadi and M. Virvou. Student modeling approaches: A literature review for the last decade. Expert Systems with Applications, 40(11):4715-4729, 2013.

[9] C. Clabaugh, G. Ragusa, F. Sha, and M. Matarić. Designing a socially assistive robot for personalized number concepts learning in preschool children. In Development and Learning and Epigenetic Robotics (ICDL-EpiRob), 2015 Joint IEEE International Conference on, pages 314-319. IEEE, 2015.

[10] H. Cuayáhuitl, M. van Otterlo, N. Dethlefs, and L. Frommberger. Machine learning for interactive systems and robots: a brief introduction. In Proceedings of the 2nd Workshop on Machine Learning for Interactive Systems: Bridging the Gap Between Perception, Action and Communication, pages 19-28. ACM, 2013.

[11] R. S. De Silva, K. Tadano, M. Higashi, A. Saito, and S. G. Lambacher. Therapeutic-assisted robot for children with autism. In Intelligent Robots and Systems, 2009. IROS 2009. IEEE/RSJ International Conference on, pages 3561-3567. IEEE, 2009.

[12] J. L. Drury, J. Scholtz, and H. A. Yanco. Awareness in human-robot interactions. In Systems, Man and Cybernetics, 2003. IEEE International Conference on, volume 1, pages 912-918. IEEE, 2003.

[13] M. Gandolfi, C. Geroin, A. Waldner, I. Maddalena, E. Dimitrova, A. Picelli, N. Smania, and C. Tomelleri. Feasibility and safety of early lower limb robot-assisted training in sub-acute stroke patients: a pilot study. European journal of physical and rehabilitation medicine, 2017.

[14] M. A. Goodrich and A. C. Schultz. Human-robot interaction: a survey. Foundations and trends in human-computer interaction, 1(3):203-275, 2007.

[15] G. Gordon, S. Spaulding, J. K. Westlund, J. J. Lee, L. Plummer, M. Martinez, M. Das, and C. Breazeal. Affective personalization of a social robot tutor for 
children's second language skills. In $A A A I$, pages 3951-3957, 2016.

[16] J. Han. Emerging technologies: Robot assisted language learning. Language Learning 85 Technology, 16(3):1-9, 2012.

[17] J. Hemminghaus and S. Kopp. Towards adaptive social behavior generation for assistive robots using reinforcement learning. In Proceedings of the 2017 ACM/IEEE International Conference on Human-Robot Interaction, pages 332-340. ACM, 2017.

[18] M. Hermann, T. Pentek, and B. Otto. Design principles for industrie 4.0 scenarios. In System Sciences (HICSS), 2016 49th Hawaii International Conference on, pages 3928-3937. IEEE, 2016.

[19] N. Jøranson, I. Pedersen, A. M. M. Rokstad, and C. Ihlebæk. Effects on symptoms of agitation and depression in persons with dementia participating in robot-assisted activity: a cluster-randomized controlled trial. Journal of the American Medical Directors Association, 16(10):867-873, 2015.

[20] P. Kan, R. Huq, J. Hoey, R. Goetschalckx, and A. Mihailidis. The development of an adaptive upper-limb stroke rehabilitation robotic system. Journal of neuroengineering and rehabilitation, 8(1):33, 2011.

[21] J. A. Kulik and J. Fletcher. Effectiveness of intelligent tutoring systems: a meta-analytic review. Review of Educational Research, 86(1):42-78, 2016.

[22] J. Lee, H. Takehashi, C. Nagai, G. Obinata, and D. Stefanov. Which robot features can stimulate better responses from children with autism in robot-assisted therapy? International Journal of Advanced Robotic Systems, 9(3):72, 2012.

[23] S. Lee, H. Noh, J. Lee, K. Lee, G. G. Lee, S. Sagong, and M. Kim. On the effectiveness of robot-assisted language learning. ReCALL, 23(01):25-58, 2011.

[24] G. Li, H. Hung, S. Whiteson, and W. B. Knox. Using informative behavior to increase engagement in the tamer framework. In Proceedings of the 2013 international conference on Autonomous agents and multi-agent systems, pages 909-916. International Foundation for Autonomous Agents and Multiagent Systems, 2013.

[25] J. B. Lyons and P. R. Havig. Transparency in a human-machine context: approaches for fostering shared awareness/intent. In International Conference on Virtual, Augmented and Mixed Reality, pages 181-190. Springer, 2014.

[26] G. Magyar and M. Vircikova. Socially-assistive emotional robot that learns from the wizard during the interaction for preventing low back pain in children. In International Conference on Social Robotics, pages 411-420. Springer, 2015.

[27] L. Ray. Cyber-physical systems. Handbook of Research on Applied Cybernetics and Systems Science, page 335, 2017.

[28] T. Salter, F. Michaud, and H. Larouche. How wild is wild? a taxonomy to characterize the 'wildness' of child-robot interaction. International Journal of Social Robotics, 2(4):405-415, 2010.
[29] B. Scassellati, H. Admoni, and M. Matarić. Robots for use in autism research. Annual review of biomedical engineering, 14:275-294, 2012.

[30] J. Scholtz. Theory and evaluation of human robot interactions. In System Sciences, 2003. Proceedings of the 36th Annual Hawaii International Conference on, pages $10-$ pp. IEEE, 2003.

[31] E. Senft, P. Baxter, J. Kennedy, and T. Belpaeme. Sparc: Supervised progressively autonomous robot competencies. In International Conference on Social Robotics, pages 603-612. Springer, 2015.

[32] F. Stroppa, C. Loconsole, S. Marcheschi, and A. Frisoli. A robot-assisted neuro-rehabilitation system for post-stroke patients' motor skill evaluation with alex exoskeleton. In Converging Clinical and Engineering Research on Neurorehabilitation II, pages 501-505. Springer, 2017.

[33] S.-H. Su, H.-C. K. Lin, C.-H. Wang, and Z.-C. Huang. Multi-modal affective computing technology design the interaction between computers and human of intelligent tutoring systems. International Journal of Online Pedagogy and Course Design (IJOPCD), 6(1):13-28, 2016.

[34] A. Tapus, C. Ţăpuş, and M. J. Matarić. User-robot personality matching and assistive robot behavior adaptation for post-stroke rehabilitation therapy. Intelligent Service Robotics, 1(2):169-183, 2008.

[35] H. A. Yanco and J. Drury. Classifying human-robot interaction: an updated taxonomy. In Systems, Man and Cybernetics, 2004 IEEE International Conference on, volume 3, pages 2841-2846. IEEE, 2004.

[36] H. A. Yanco and J. L. Drury. A taxonomy for human-robot interaction. In Proceedings of the AAAI Fall Symposium on Human-Robot Interaction, pages 111-119, 2002.

[37] L. Zollo, K. Wada, and H. M. Van der Loos. Special issue on assistive robotics [from the guest editors]. IEEE Robotics \& A Atomation Magazine, 20(1):16-19, 2013. 